\title{
Echocardiographic pattern and severity of valve dysfunction in children with rheumatic heart disease seen at Uganda Heart Institute, Mulago hospital.
}

\author{
Sulaiman Lubega ${ }^{1}$, Twalib Aliku, ${ }^{1,2}$, Peter Lwabi ${ }^{1}$
}

1. Uganda Heart Institute, Mulago hospital complex, Kampala:

2. Uganda Heart Institute, Gulu University

\begin{abstract}
:
Background: Rheumatic heart disease (RHD) is the commonest acquired heart disease in children worldwide but in Uganda, data is scarce regarding its morbidity and mortality. The disease has a progressive course and patients usually require valve repair/replacement in the future.

Objectives:

To describe the frequency of echocardiographic valvular dysfunction in children with RHD

To explore the relationship between the severity of valvular dysfunction by the age and sex of the children with RHD

Methods: Echocardiographic findings of children $\leq 15$ years with RHD seen at Uganda Heart Institute from January 2007 to December 2011 were retrospectively analyzed.

Results: 376 children had a diagnosis of RHD. The mean age of the children was $11.0 \pm 2.7$ years and $216(57.4 \%)$ were females. Mitral regurgitation was the commonest lesion seen in $98.9 \%$ (severe in $73.1 \%$ ) of the children. Aortic regurgitation (AR) was found in 51.3\% (severe in 7.2\%), mitral stenosis (MS) was found in 10.6\% (severe in 5.9\%), tricuspid regurgitation was found in $86.7 \%$ (severe in $8.2 \%$ ) while aortic stenosis was seen in $1.3 \%$ (severe in $0.3 \%$ ). Severe AR was less common in females $(\mathrm{OR}=0.32,95 \% \mathrm{CI} 0.13-0.78)$ and children with MS were older than those without MS $(12.7 \pm 2.0$ Vs. $10.7 \pm 2.7$ years, $\mathrm{p}<0.00)$.

Conclusions: Mitral valve dysfunction was found in almost all the cases of RHD and majority of the children had severe valve disease at the time of their first presentations. Children with MS were predominantly above 10 years and severe AR was more common in males.
\end{abstract}

Keywords: Rheumatic heart disease, Valvular dysfunction

DOI: http://dx.doi.org/10.4314/ahs.v14i3.17

\section{Introduction:}

Rheumatic heart disease (RHD) is a major cause of cardiac morbidity and mortality among children in the developing countries. About 15 million cases of RHD are reported worldwide, with 282,000 new cases and 233,000 deaths annually.1

Rheumatic heart disease begins in childhood, has a progressive course and patients usually require valve repair/ replacement in future, which is not routinely available in many sub-Saharan countries including Uganda. Pro

\section{Corresponding author: \\ Lubega S \\ (Paediatrics) Uganda Heart Institute, Mulago Hospital complex, \\ P.O BOX 7051, Kampala. \\ Tel. +256-772-493960, \\ Email: lubsk2001@yahoo.com}

phylactic penicillin against recurrent rheumatic fever is paramount in preventing progression from early to severe RHD.2,3

There are very few published studies 4,5 regarding valvular dysfunction in children with RHD in the Sub-Saharan region of Africa which has the world's highest prevalence of RHD (15-20 per 1000 people). 6

Most of the publications 7-12 so far have been from adults studies that also included a small proportion of children. These studies have shown mitral regurgitation (MR) as the commonest valvular dysfunction with a prevalence of $56-96 \%$, mitral stenosis (MS) occurring in $20-50 \%$, aortic regurgitation (AR) in $19-41 \%$, aortic stenosis (AS) in 1-9\%, tricuspid regurgitation (TR) in $21-40 \%$, with majority of the patients having mixed valvular dysfunction. These studies have also shown that rheumatic mitral valve disease is related to age with patients below 20 years having a predominance of MR, those 20-40 years having a predominance of MS, while 
those above 40 years having a fair mix of MR and MS.

There was no school screening program for rheumatic heart disease/rheumatic fever in place during the study period; however, a survey done between August and November 2010 in primary school children (5 to 16 years of age) in Kampala district by Beaton A. et al4 found 72 out of 4,869 to have RHD resulting in a prevalence rate of 14.8 cases per 1000 children $(95 \%$ confidence interval, 7.3-22.3). Most of these cases $(97.8 \%)$ had mitral valve disease only.

\section{This study aimed at:}

Describing frequency of echocardiographic valvular dysfunction in children with RHD

Exploring the relationship between the severity of valvular dysfunction by the age and sex of the children with RHD.

\section{Methodology:}

This was a retrospective descriptive study done at the Uganda Heart Institute located in Mulago Hospital Complex, which is the National referral hospital of Uganda. Our institute being a super-specialized centre, almost all the patients seen are referred from lower health units. The hospital does not have an active heart valve surgery programme and balloon mitral valvotomy procedures are not routinely done. These procedurs are occasionally done with visiting foreign missions.

All echocardiography reports of children 15 years and below with a diagnosis of RHD done from January 2007 to December 2011 were retrieved from the computer records.

Information obtained included age, gender, indications for referral, and echocardiographic findings.

The addresses of the patients were not routinely included in the available records.

Children were recruited only once in the 5 years of the study period and we considered the initial echocardiography report for cases screened more than once. $\mathrm{Pa}$ tients who had received heart valve surgery at the initial visit were excluded.

Trans-thoracic echocardiographies were done by 2 paediatric cardiologists using Hp Sonos 4500 and Sonos 5500 ultrasound machines, and the diagnosis and interpretation of valvular dysfunction was based on the ACC/AHA 200613 Guidelines for the management of patients with valvular heart disease and the American society of echocardiography report.14 The following aspects of the guidelines were used for grading valvular dysfunction severity: For mitral and tricuspid regurgitation we used regurgitant jet area, regurgitant fraction, and corresponding heart chamber size. For aortic and pulmonary regurgitation we used regurgitant jet area, regurgitant flow deceleration slope, and corresponding heart chamber size. For mitral, aortic and tricuspid valve stenosis we used valve orifice area by planimetry, forward flow mean pressure gradient and pre-stenotic heart chamber size.

Data was summarized in frequency tables and pie charts. Relationships between continuous and categorical variables were assessed using Student's T-test and Chi- Square test consecutively, except where otherwise indicated.

Permission to carry out the research and waiver of consent to review the records of patients were obtained from the relevant research and ethics committees.

\section{Results:}

There were 3,503 abnormal echo reports of children 15 years and below that were reviewed: 2,677 of these had congenital heart diseases while 826 had acquired heart diseases. Of all those with acquired heart diseases, $376(45.5 \%)$ had RHD, 22.4\% had dilated cardiomyopathy, $8.8 \%$ had pericarditis,

$7.9 \%$ had Cor pulmonale, $7.1 \%$ had endomyocardial fibrosis, $1.9 \%$ had hypertensive heart disease, $1.3 \%$ had heart tumors, $0.4 \%$ had infective endocarditis, and $4.6 \%$ had cardiomegaly with hyperdynamic heart state (commonly in patients with sickle cell disease).

Of the children with RHD, 216 (57.4\%) were females. The mean age $( \pm S D)$ of the children with RHD was 11.0 \pm 2.7 years (Range 4 to 15 years).

Out of the 376 cases, $269(71.5 \%)$ had the reasons for referral indicated on the echocardiography reports. Of these cases with indications, $44.2 \%$ were due to suspected RHD, $27.9 \%$ due to features of CCF, $17.5 \%$ due to heart murmur, $3.0 \%$ due to palpitations, $2.6 \%$ due to suspected infective endocarditis, $2.6 \%$ due to cardiomegaly on CXR, while rare indications like arrhythmias, syncope, and chest pain accounted for the remaining $2.2 \%$ 
Prevalence and severity of valvular dysfunction

All except one of the 376 cases studied had mitral valve disease (MR or MS).

Table 1a shows that out of the 376 children studied, MR was the commonest lesion seen in

$98.9 \%$ of all cases studied and it accounted for 36.8\%

of the total valvular lesions, while TS was the

least common valvular lesion found in $0.3 \%$ of all the cases and $0.1 \%$ of the total valvular lesion.

Majority of patients had more than one valvular lesions and this is depicted by the total of the

percentage of valvular lesions being 269\%; implying that each patient had an average of about 2.69

valve lesions.

Mitral regurgitation was the most prevalent severe valvular lesion found in $73.1 \%$ of all cases and there was

Table 1a: Frequencies of Valvular dysfunction among the study participants $(N=376)$

\section{Valvular lesions}

\section{Valve dysfunction}

$\mathbf{n}$

\section{Percentage of total valvular lesions}

Percent of all cases

\begin{tabular}{cccc}
\hline MR & 372 & $36.8 \%$ & $98.9 \%$ \\
MS & 40 & $4.0 \%$ & $10.6 \%$ \\
AR & 193 & $19.1 \%$ & $51.3 \%$ \\
AS & 5 & $0.5 \%$ & $1.3 \%$ \\
TR & 326 & $32.2 \%$ & $86.7 \%$ \\
TS & 1 & $0.1 \%$ & $0.3 \%$ \\
PR & 75 & $7.4 \%$ & $19.9 \%$ \\
\hline TOTAL & 1012 & $100.0 \%$ & $269.1 \%{ }^{\#}$ \\
\hline
\end{tabular}

MR Mitral Regurgitation, TR Tricuspid Regurgitation, AR Aortic Regurgitation, PR Pulmonary regurgitation, MS Mitral Stenosis, AS Aortic Stenosis, TS Tricuspid Stenosis,

\#

more that $100 \%$ because some patients had more than one valve lesion 
no case of severe tricuspid stenosis encountered as depicted by table $1 \mathrm{~b}$.
Of all the cases studied, $301(80 \%)$ had severe dysfunction of at least one of the heart valves, majority of

Table 1b: Grading of Valvular dysfunction among all the cases studied $(\mathrm{N}=376)$

\section{Severe,}

Valve dysfunction
Moderate,

n $(\%)$

$59(15.7)$

$9(2.4)$

$44(11.7)$

$3(0.8)$

$70(18.6)$

$1(0.3)$
Mild,

n $(\%)$

$38(10.1)$

$9(2.4)$

$122(32.4)$

$1(0.3)$

$225(59.8)$

O (0) PR $0(0)$

$5(1.3)$

$70(18.6)$

MR Mitral Regurgitation, TR Tricuspid Regurgitation, AR Aortic Regurgitation,,

PR Pulmonary regurgitation, MS Mitral Stenosis, AS Aortic Stenosis, TS Tricuspid Stenosis

\# This is more than 100\% because some patients had more than one valve lesion

whom had severe MR.

Figure 1 shows the co-existence of the 3 most prevalent valvular lesions. One hundred and sixty-eight children had a combination of MR, AR and TR, 191 had MR with AR, 322 had MR with TR, while 170 had AR with TR.

There were additional combinations of regurgitation and stenosis not shown in the venn diagram. Majority

\section{Figure 1: Multi-Valvular dysfunction}

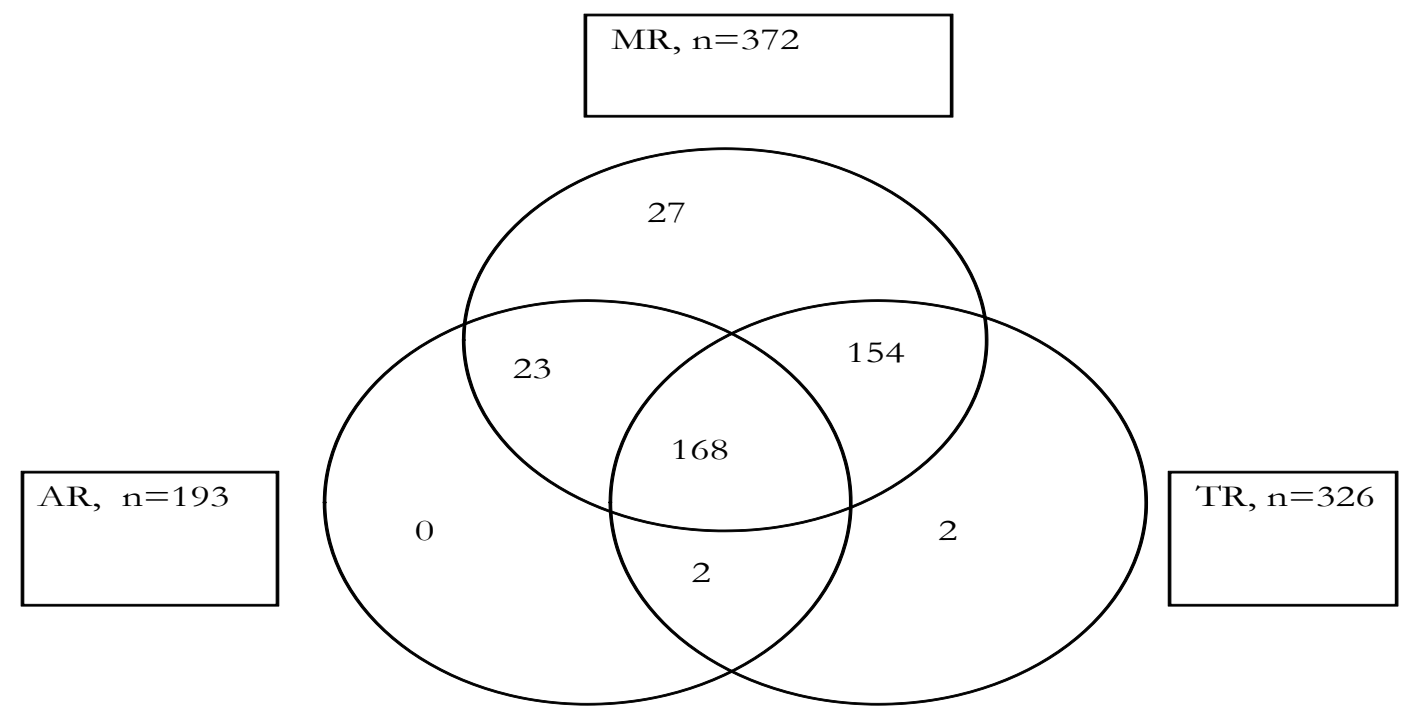


of the children (37 out of 40) children with MS had concomitant MR; all the 5 children with AS had AR and MR; and the single child with TS also had TR, MR and AR.

Figure 2 shows that 21 children had severe MR with severe AR, 29 had severe MR with severe TR, and 2 had severe AR with severe TR, while 2 children had a com- bination of severe MR, severe $\mathrm{AR}$ and severe TR. Of the 22 children with evere MS (not shown in the venn diagram), 4 had severe MR, 3 had severe TR but none of them had severe AR or severe AS. The single child with severe AS also had moderate AR.

The severity of TR mirrored that of pulmonary artery hypertension (PHT), p value $<0.001$ (by multinominal

Figure 2: Severe Multi-Valvular dysfunction

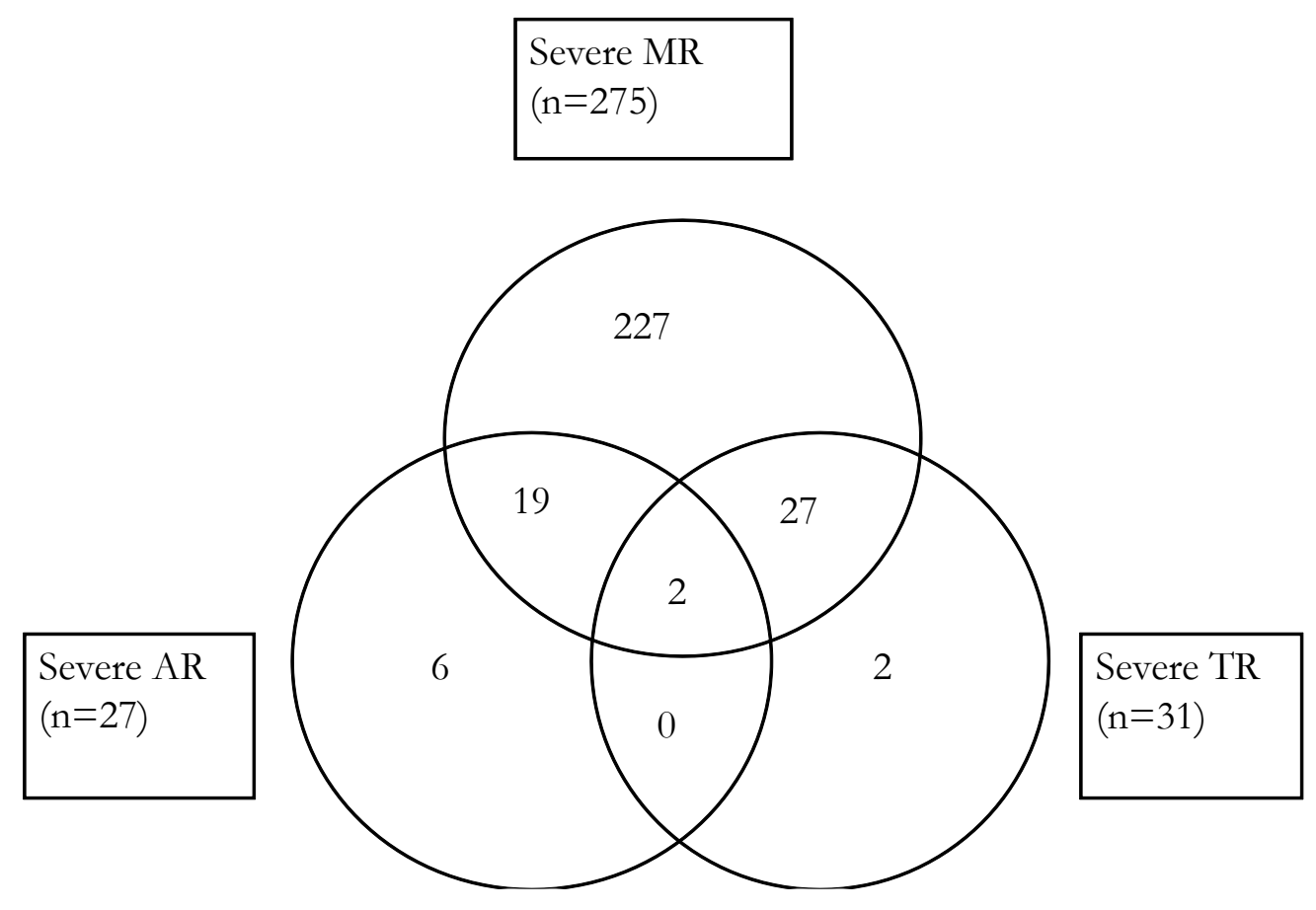

logistic regression and chi square tests).

\section{Sex and age versus valvular severity}

Females were 1.6 times more likely to have severe MR than males but this was of borderline significance $(p$ value $=0.05)$ (Table $2 \mathrm{a}$ ), while on the other hand being female was protective against having severe AR ( $p$ value $=0.01)$ (Table 2b).

There was no gender difference associated with the severity of TR ( $p$ value $=0.27$ ) or MS ( $p$ value $=0.77$ ). 
Mitral regurgitation

Demographics

$\begin{array}{lll}\text { Severe }(\mathrm{n}=275) & \text { Mild-Mod }(\mathrm{n}=97) & \text { OR }(\mathrm{CI})\end{array}$
Age (Yrs), Mean \pm SD
$10.84 \pm 2.69$
$11.28 \pm 2.81$

Age category

$$
\begin{aligned}
& <10 \text { yrs } \\
& \geq 10 \text { yrs }
\end{aligned}
$$

$84(30.5 \%)$

$25(25.8 \%)$

$1.26(0.75-2.15)$

$191(69.5 \%)$

$72(74.2 \%)$

Sex

Female

$167(60.7 \%)$

$48(49.5 \%)$

$1.58(0.99-2.52)$

Male

$108(39.3 \%)$

$49(50.5 \%)$

OR (CI) Odds Ratio (Confidence Interval), n number

There was no significant age difference between children with mild-moderate MS and those with severe MS ( $p$ value $=0.24)$ but that the mean age of children with any degree of MS (12.7 \pm 2.0 years) was significantly higher than that of children without MS $(10.7 \pm 2.7$ years) ( $p$ value $<0.001$ ).

Age did not influence the severity of $M R(p$ value $=0.18)$, AR $(\mathrm{p}$ value $=0.33)$ or TR $(\mathrm{p}$ value $=0.91)$.

\section{Other findings:}

\section{Vegetations:}

Fourteen (14) out of the 376 children (3.7\%) had visible vegetations on heart valves; 12 on mitral valve, 1 on aortic valve and 1 on tricuspid Valve. Three children had large vegetations $(\geq 10 \mathrm{~mm}$ in diameter) on mitral valves and associated severe MR.

\section{Left ventricular systolic dysfunction}

Left ventricular (LV) systolic dysfunction (LV fractional shortening $<25 \%$ using $\mathrm{M}$ mode measurements at mitral valve tip in parasternal long axis) was found in 19 cases, all with mitral valve disease $(68.4 \%$ of them with severe MR).

\section{Pulmonary artery hypertension}

Pulmonary hypertension (measured indirectly by TR Pressure gradient $>30 \mathrm{mmHg}$ in absence of right ventricular outflow tract obstruction) was found in 291 $(77.4 \%)$ of the 376 children studied.

\section{Discussion:}

There is general scarcity of published data related to childhood RHD in the African countries that are most affected by the disease. This study aimed to describe 
Aortic regurgitation
Demographics
Severe $(n=27)$
Mild-Mod ( $\mathrm{n}=166)$
$\mathrm{OR}(\mathrm{CI})$

Age (Yrs), Mean $\pm S D$

$11.48 \pm 2.74$

$10.92 \pm 2.80$

\begin{tabular}{|c|c|c|c|c|}
\hline \multirow[t]{2}{*}{ Age category } & $<10 \mathrm{yrs}$ & $7(25.9 \%)$ & $49(29.5 \%)$ & $0.83(0.33-2.10)$ \\
\hline & $\geq 10$ yrs & $20(74.1 \%)$ & $117(70.5 \%)$ & \\
\hline \multirow[t]{2}{*}{ Sex } & Female & $8(29.6 \%)$ & $94(56.6 \%)$ & $0.32(0.13-0.78)$ \\
\hline & Male & $19(70.4 \%)$ & $72(43.4 \%)$ & \\
\hline
\end{tabular}

OR (CI) Odds Ratio (Confidence Interval), n number

frequency of rheumatic valvular dysfunction and to explore the relationship between the severity of valvular dysfunction by the age and sex of the children with RHD

\section{Prevalence of valvular dysfunction}

All except one of the 376 cases studied had mitral valve disease ( 372 had MR while 3 had MS). This finding is similar to what was also reported in Nigeria7 and Pakistan8 in which $97-100 \%$ of all patients with RHD had mitral valve disease. The high prevalence of $\mathrm{MR}$ in this study of $98.6 \%$ is also comparable to $80-98 \%$ reported in some of the previous studies done in Kenya5, Khartoum15 and most recently in a school survey in Uganda4. Apart from the Ugandan study that included only children less than 16 yrs, the other studies included all age groups from 5 to 90 years. In a South African RHD registry 9 of patients 15 years and above, MR was found to be the predominant valve lesion implying that this pattern is carried on into adulthood. Mitral regurgitation has, however, also been reported to occur in as low as 37\% of cases of RHD in an isolated large retrospective study done in India16 of children up to 18 years.
Previous studies 7, 8, 10,11,15,16 have reported AR to occur in $20-41 \%$ of RHD cases which is lower than what was found in the current study of $51 \%$. We could not find a clear explanation for this disparity but again the fact that majority of our study population had severe valve dysfunction cannot be ignored.

The prevalence of MS in the current study of $11 \%$ is lower than the $20-50 \%$ from previous studies.7-12, 16 This is most likely due to the fact these previous studies had a significantly higher proportion of adults in whom rheumatic MS has been found to be more prevalent.

The prevalence of TR of $87 \%$ in the current study is much higher than the $22-48 \%$ found previously.7, 8,10 , 16 Majority of the TR was functional TR resulting from pulmonary hypertension (PHT) which is a known complication of severe valvular heart disease.17, 18

The high prevalence of severe valvular disease $(80 \%)$ and PHT (77.4\%) in this study lends support to this argument.

Majority of the children had mixed valve lesion involving various combinations of MR, TR, AR and MS just like in previous studies. 7, 8, 10-12, 15 


\section{Severity of valvular dysfunction}

Although all cases of RHD were found to be mild and asymptomatic in a survey of school children in Uganda by Beaton et al4, majority ( $\mathrm{n}=301,80 \%$ ) of the children in our study presented

with at least one severe valvular lesion, and MR was the most prevalent of these (275 out of 301$)$. The results of this study could suggest that once children with RHD are symptomatic of severe valvular disease they stop attending school. Our institution is a national referral Centre and children could have presented late either because of delayed disease identification and/or barriers to referral of cases. All this re-emphasizes the need for effective and accurate ways to detect children with early RHD when secondary prophylaxis may be of greatest benefit.2,3

Majority of the children with MS had concomitant MR of any degree but out of the 22 children with severe MS, only 4 had severe MR (OR=0.13, $\mathrm{CI}=0.03-0.58)$. Although the number of cases was small, it was found that children with severe MS were unlikely to have severe $\mathrm{MR}$, a finding that has also been reported earlier.16 Of the 22 children with severe MS in this study, 8 had concomitant moderate-severe MR rendering catheter based balloon mitral valvulotomy out of the treatment options.

All the children with severe TR in this study had other concomitant severe valvular lesions (either MR, MS or AR) and additionally the severity of TR was significantly related to that pulmonary hypertension $(\mathrm{p}<0.001)$. All these support the notion that tricuspid regurgitation is usually secondary to the pulmonary hypertension that complicates RHD17, 18 as explained earlier.

Sex and age versus valvular severity

Female were less likely to have severe AR, OR $=0.32$ $(95 \% \mathrm{CI}=0.13-0.78)$ but the small number of children with severe AR in this study limits the importance of this finding. A study done in Turkey19 showed that males were more likely to have severe AR, but another study in Pakistan 8 showed the reverse with AR being more frequent in females.

Children with MS were significantly older than those without MS in this study (mean age

12.7.0 \pm 2.0 Vs $10.7 \pm 2.7$ years, $\mathrm{P}<0.001)$. Similarly, studies9,12,16 that have looked at all age groups with RHD have also found MS to be more prevalent above
10 years of age.

\section{Conclusions:}

Mitral valve dysfunction was the most prevalent valvular lesion and majority of the children studied had mixed valve lesions.

Majority of the cases had already progressed to severe valvular dysfunction at the time of their first presentations at the institute.

Children with MS were significantly older than those without MS and severe AR was found to be less prevalent among females.

\section{Recommendations:}

There is need to study the reasons for late presentation of children with RHD to our national referral centre.

There is a need to re-study the association of severe AR and gender among children with AR as the predominant lesion of the RHD.

\section{Limitations:}

This was a cross sectional survey of children referred to a tertiary hospital in Uganda. This being hospital data, the results may not reflect the true prevalence of the valve lesions in the community. A number of factors could have influenced the profile of children reaching the hospital leading to bias.

The echocardiography images were not reviewed in this study and so the accuracy of assessment of valve dysfunction could not be assessed.

Valvular pathology except valve area by planimetry was not captured from the patient records.

\section{Disclosure:}

None

\section{Acknowledgements:}

Miss Jane Atugonza helped with data entry and Dr. Eric Wobudeya

(Epidemiologist/Paediatrician) helped with Data analysis.

References:

1. Carapetis JR, Steer AC, Mulholland EK, Weber M. The global burden of group A streptococcal diseases. 
Lancet Infect Dis. 2005;5:685-694.

2. Majeed HA, Batnager S, Yousof AM, et al. Acute rheumatic fever and the evolution of rheumatic heart disease: a prospective 12 year follow-up report. Journal of clinical epidemiology. 1992;45:871-5.

3. Feinstein, A. R. et al. Rheumatic fever in children and adolescents. A long-term epidemiologic study of subsequent prophylaxis, streptococcal infections, and clinical sequelae.. Ann. Intern. Med.1964; 68-86: 60 (Suppl. 5). 4. Beaton A. Okello E, Lwabi P. et al. Echocardiography screening for rheumatic heart disease in Ugandan schoolchildren. Circulation. 2012 Jun 26;125(25):31273132.

5. Yuko-Jowi C, Bakari M. Echocardiographic patterns of juvenile rheumatic heart disease at the Kenyatta National Hospital, Nairobi. . East Afr Med J. 2005 Oct;82(10):514-9.

6. WHO Regional Committee for Africa. Cardiovascular diseases in the African region: current situation and perspectives-report of the regional director 2005. Available online: http://www.afro.who.int/rc55/documents/afr_rc55_12_cardiovascular.pdf

7. Sani MU, Karaye KM, Borodo MM. Prevalence and pattern of rheumatic heart disease in the Nigerian savannah: an echocardiographic study. Cardiovasc J Afr. 2007 Sep-Oct;18(5):295-9.

8. Aurakzai HA, Hameed S, Shahbaz A, et al. Echocardiographic profile of rheumatic heart disease at a tertiary cardiac centre. J Ayub Med Coll Abbottabad. 2009 Jul-Sep;21(3):122-6.

9. Karen Sliwa, Melinda Carrington, Bongani M. Mayosi et al. Incidence and characteristics of newly diagnosed rheumatic heart disease in Urban African adults: insights from the Heart of Soweto Study: Eur Heart J (2010) 31 (6): 719-

727.

10. Tantchou Tchoumi JC, Butera G. Rheumatic valvulopathies occurence, pattern and follow-up in rural area: the experience of the Shisong Hospital, Cameroon. Bull Soc Pathol Exot. 2009 Aug;102(3):155-8.
11. Essien IO, Onwubere BJ, Anisiuba BC, et al. One year echocardiographic study of rheumatic heart disease at Enugu, Nigeria. Niger Postgrad Med J. 2008 Sep;15(3):175-8.

12. Saleh HK. Pattern of Rheumatic heart disease in Southern Yemen. Saudi Med J.

2007 Jan;28(1):108-13.

13. ACC/AHA 2006 Guidelines for the Management of Patients with Valvular Heart Disease. A Report of the American College of Cardiology/American Heart Association Task Force on Practice Guidelines J Am Coll Cardiol 2006; 48: e1148.

14. Recommendations for Evaluation of the Severity of Native Valvular Regurgitation with Two-dimensional and Doppler Echocardiography. American society of echocardiography report. J Am Soc echocardiogr 2003; 16: 777-802

15. Alkhalifa MS, Ibrahim SA, Osman SH. Pattern and severity of rheumatic valvular lesions in children in Khartoum, Sudan. East Mediterr Health J. 2008 SepOct;14(5):1015-21.

16. Chockalingam A, Gnanavelu G, Elangovan S, et al. Clinical spectrum of chronic rheumatic heart disease in India. J Heart Valve Dis. 2003 Sep;12(5):577-81.

17. Fawzy ME, Mimish L, Sivanandam V, et al. Immediate and long-term effect of mitral balloon valvotomy on severe pulmonary hypertension in patients with mitral stenosis. Am Heart J. 1996; 131: 89-93.

18. Skudicky D, Essop MR, Sareli P. Efficacy of mitral balloon valvotomy in reducing the severity of associated tricuspid valve regurgitation. Am J Cardiol. 1994; 73: 209-11.

19. Ozer O, Davutoglu V, Sari I, Akkoyun DC, Sucu M. The spectrum of rheumatic heart disease in the southeastern Anatolia endemic region: results from $1900 \mathrm{pa}-$ tients. J Heart Valve Dis. 2009 Jan;18(1):68-72. 